\title{
Magnetostatic Coupling Between two Nanowires of Different Width
}

\author{
Hanseok Lee ${ }^{1}$, Seungho Kim ${ }^{1}$, Youngwook Chang ${ }^{1}$, Kyung-Hwa Yoo ${ }^{1}$, and J. Lee ${ }^{1 *}$ \\ ${ }^{1}$ Institute of Physics and Applied Physics, Yonsei University, Seoul 120-749, Korea
}

(Received 3 November 2008, Received in final form 20 February 2009, Accepted 11 March 2009)

\begin{abstract}
The magnetostatic interaction between the two magnetic nanowires was studied by using the longitudinal magneto-optical Kerr effect (MOKE). For this purpose two magnetic nanowires having different widths (400 nm, $800 \mathrm{~nm}$ ) were fabricated on an Si substrate with electron beam lithography and the lift-off method. Magnetic hysteresis loops measured by MOKE showed double switching behavior, corresponding to the separated switching fields of each wire. The switching field of the narrow wire was greatly affected by the separation between the two wires. Based on how the switching field changes with decreasing separation, it is concluded that the magnetostatic field of the 800-nm wire strongly affects the switching of the 400-nm wire when the separation is less than $0.5 \mu \mathrm{m}$.
\end{abstract}

Keywords : nanowire, anisotropy, magnetostatic interaction, MOKE

\section{Introduction}

Ferromagnetic nanowires have been studied extensively over the last decade [1]. The wires have a well-defined magnetic anisotropy because of their shape, and many fascinating properties and behaviors [2-11], including single domain structure, domain wall motion along the wire axis, spin transfer torque, and use in magnetic logic devices, have their roots in this well-defined anisotropy.

The magnetic hysteresis loop is one of the most important measurements used in understanding magnetic materials, and it is directly affected by the magnetic anisotropy. Much of what is known about magnetic nanostructures is based on the measurements of magnetic hysteresis loops, but it is necessary to study the magnetostatic interaction among multiple magnetic nanostructures in order to understand the hysteresis loop of a nanostructure array.

Recently, there have been several reports about the magnetic dipolar interaction in magnetic nanowire arrays. The coercive field of a nanowire array has been found to be influenced strongly by the edge-to-edge spacing between the nanowires [12]. This variation in the coercive field has also been observed in a wire array of alternating widths [13]. However, in order to understand the mag-

*Corresponding author: Tel: +82-2-2123-4004

Fax:+82-2-363-4004, e-mail: j110017@phya.yonsei.co.kr netic dipolar interaction better, it is necessary to study the interaction between two wires only, particularly given the fact that a pair of wires is the basic unit in forming a wire array.

In this work, we report details about the magnetostatic interaction between two magnetic nanowires. For our study we used two wires with different widths and with different separations between them. We explain our observations in terms of the magnetostatic field from the wires.

\section{Experiment}

Six pairs of nanowires $\left(10 \mathrm{~nm} \mathrm{Cu}, 20 \mathrm{~nm} \mathrm{Ni}{ }_{80} \mathrm{Fe}_{20}\right.$, and $3 \mathrm{~nm} \mathrm{Cu}$ capping) were prepared on a $\mathrm{Si}$ substrate with electron beam lithography and the lift-off method. Each pair was composed of two nanowires with different widths (400 $\mathrm{nm}$ and $800 \mathrm{~nm}$ ). The separation $(s)$ between two wires in a pair ranged from $0.07 \mu \mathrm{m}$ to $2 \mu \mathrm{m}$. The length of the wires was $100 \mu \mathrm{m}$. Each pair was separated from adjacent pairs by $10 \mu \mathrm{m}$, which is a large enough distance that the magnetostatic interaction between the pairs can be neglected. In this paper we discuss only pairs with separations $\mathrm{s}=0.07 \mu \mathrm{m}$ and $\mathrm{s}=2 \mu \mathrm{m}$. The evaporation step was done under the low pressure of $10^{-8}$ Torr. Fig. 1 shows an atomic force microscopy (AFM) image of two nanowire pairs.

We used the longitudinal magneto optical Kerr effect (MOKE) to detect the magnetic hysteresis loop of the 


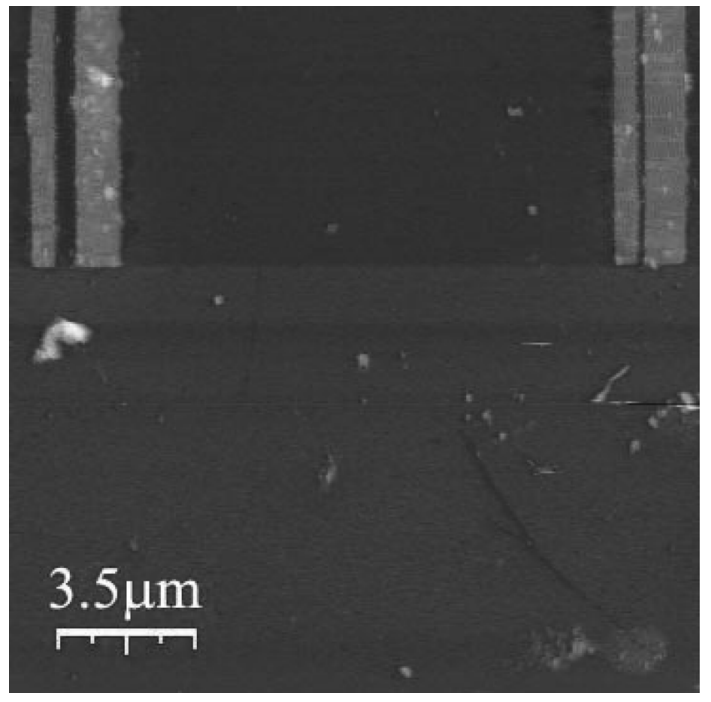

Fig. 1. Atomic force microscopy image of two nanowire pairs; the separations between the two wires are $0.2 \mu \mathrm{m}$ (left) and $0.07 \mu \mathrm{m}$ (right).

wires. Fig. 2(a) shows the MOKE setup for this experiment. The s-polarized light (of wavelength $633 \mathrm{~nm}$ ) was incident at $45^{\circ}$ to the surface normal, and the reflected beam was detected by the photodiode. The incident beam was focused onto a spot by using an objective lens $(50 \times)$ with a long working distance $(17 \mathrm{~mm})$. We confirmed that

(a)

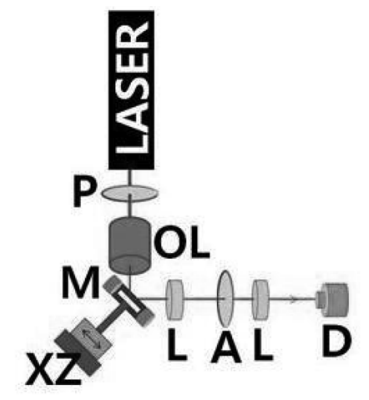

(b)

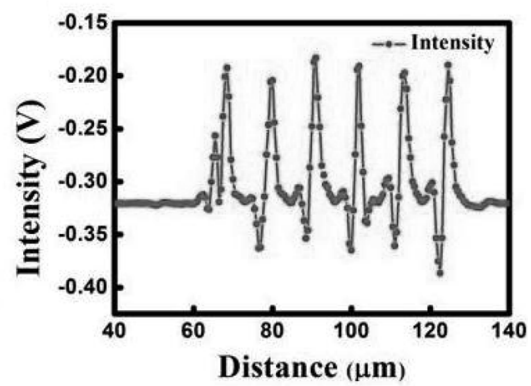

Fig. 2. (a) Experimental setup for MOKE measurements (LASER: HeNe laser, P: polarizer, OL: objective lens, M: magnet, XZ: XZ-motional stage, A: analyzer, L: lens, D: detector). (b) Intensity of the reflected beam from the nanowire pair. The separations between the two wires are (from left to right) $2,1,0.5,0.2,0.1,0.07 \mu \mathrm{m}$. the focused beam covered just one pair of nanowires by measuring the intensity of the reflected beam from the pairs. During measurements we scanned the sample along one direction by using an xz-motion stage ( $0.5 \mu \mathrm{m}$ step). The results of these intensity measurements are shown in Fig. 2(b). Six main peaks can be seen at the positions where the pairs are located. The separation between two intensity maxima is $11-12 \mu \mathrm{m}$, which is the same as the distance between the nanowire pairs. The peak is partly split into two peaks for the nanowire pair with a separation of $2 \mu \mathrm{m}$. From this measurement it is clear that the focused beam is small enough to detect the signal from a single pair only.

\section{Results and Discussion}

Fig. 3 shows the hysteresis loops of the two pairs of nanowires having separations $s=2 \mu \mathrm{m}$ and $s=0.07 \mu \mathrm{m}$. Both loops show a very sharp double switching behavior. Since the switching field of the nanowire increases as the width of the wire decreases, the smaller of the two switching fields is the switching field of the $800-\mathrm{nm}$ wire $\left(H_{s w 800}\right)$ while the larger is the switching field of the 400nm wire $\left(H_{s w 400}\right)$.

Since the focused beam covers a single nanowire pair, the hysteresis loop represents the sum of the orientations of the two magnetizations in a pair. Assume that both wires are saturated by a sufficiently large negative field (1). As long as the positive applied field $\left(H_{\text {app }}\right)$ is smaller than $H_{s w 800}$, the two magnetizations will remain parallel to each other. As $H_{a p p}$ increases, the magnetization of the 800-nm wire switches at $H_{s w 800}$, causing the two magnetizations to be in antiparallel alignment (2). As $H_{a p p}$ is increased further and becomes larger than $H_{s w 400}$, the the

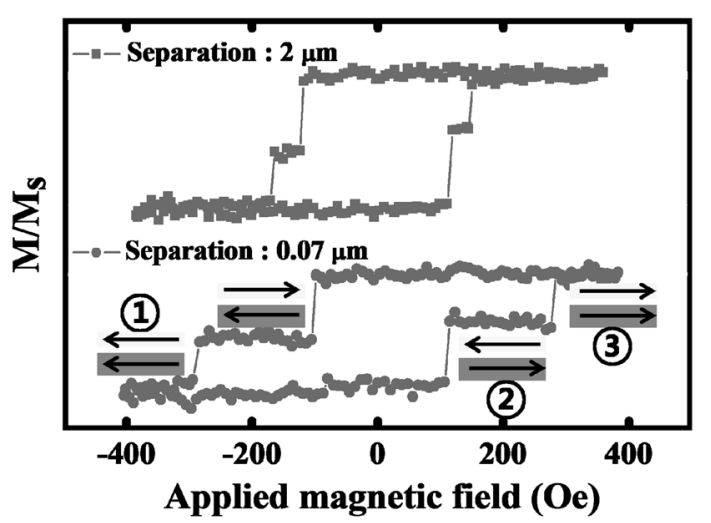

Fig. 3. Magnetic hysteresis loops of two nanowire pairs with the magnetic field applied parallel to the wire axis. The separation in each pair of wires is shown. (Arrows indicate the direction of the magnetization in each wire.) 
magnetization of the 400-nm wire is reversed, and the two magnetizations are once again in parallel alignment (3). During the negative field sweep, the same thing occurs.

Comparing the two hysteresis loops, we can see that $H_{s w 400}(284 \mathrm{Oe})$ for the pair with $0.07-\mu \mathrm{m}$ separation increases much more than $H_{s w 400}(158$ Oe) for the pair with 2- $\mu \mathrm{m}$ separation, while $H_{s w 800}$ decreases slightly less for the the pair with $0.07-\mu \mathrm{m}$ separation than for the pair with $2-\mu \mathrm{m}$ separation $(116 \mathrm{Oe} \rightarrow 101 \mathrm{Oe})$. The variation of the switching field can be observed when the separation is smaller than $0.5 \mu \mathrm{m}$.

The switching field variation can be understood in terms of the magnetostatic field from each wire. In the case of the magnetostatic field from the parallel magnetization state (1) there is a residual field from the $400 \mathrm{~nm}$ wire that points along the same direction as the applied field. Therefore $H_{s w 800}$ is smaller than it would be without the presence of this magnetostatic field. In the case of the antiparallel magnetization state (2) there is magnetostatic field from the 800-nm wire that points in a direction opposite to $H_{a p p}$ at the position of the $400-\mathrm{nm}$ wire. In order to reverse the magnetization of the $400-\mathrm{nm}$ wire, $H_{\text {app }}$ must overcome this extra field. Therefore $H_{s w 400}$ is larger than it would be without the magnetostatic field. Since magnetostatic fields are stronger at small separation, the effect of the magnetostatic field is stronger for $s$ $=0.07 \mu \mathrm{m}$ than for $s=2 \mu \mathrm{m}$. At a given separation, the strength of the magnetostatic field is proportional to the magnetic moment within the wire, which implies that the magnetostatic field from an $800-\mathrm{nm}$ wire is stronger than that from a $400-\mathrm{nm}$ wire. Therefore the effect of the interaction becomes more prominent in $H_{s w 400}$ than in $H_{s w 800}$.

\section{Conclusion}

We have measured the magnetic hysteresis loops of $\mathrm{Ni}_{80} \mathrm{Fe}_{20}$ nanowire pairs by using highly sensitive longi- tudinal MOKE. We observed a strong magnetostatic interaction between two magnetic nanowires with a separation $s=0.07 \mu \mathrm{m}$, and this magnetostatic interaction was reflected in the increased switching field of the 400-nm wire. This can be explained by the existence of the magnetostatic field from the $800-\mathrm{nm}$ wire. We did not observe changes in the switching field for separations $s>0.5 \mu \mathrm{m}$.

\section{References}

[1] J. I. Martin, J. Nogues, K. Liu, J. L. Vincenr, and I. Schuller, J. Magn. Magn. Mater., 256, 449 (2003), and rereferences therein.

[2] T. Ono, H. Miyajima, K. Shigeto, K. Mibu, N. Hosoito, and T. Shinjo, Science 284, 468 (1999).

[3] D. Atkinson, D. A. Allwood, G. Xiong, M. D. Cooke, C. C. Faulkner, and R. P. Cowburn, Nat. Mater. 2, 85 (2003).

[4] G. S. D. Beach, C. Nistor, C. Knutson, M. Tsoi, and J. L. Erskine, Nat. Mater. 4, 741 (2005).

[5] G. S. D. Beach, C. Knutson, C. Nistor, M. Tsoi, and J. L. Erskine, Phys. Rev. Lett. 97, 057203 (2006).

[6] M. Hayashi, L. Thomas, Ya. C. Rettner, R. Moriya, Y. B. Bazaliy, and S. S. P. Parkin, Phys. Rev. Lett. 98, 037204 (2007).

[7] D. A. Allwood, G. Xiong, C. C. Faulkner, D. Atkinson, D. Petit, and R. P. Cowburn, Science 309, 1688 (2005).

[8] S. S. P. Parkin, U.S. Patent No. 6834005 (2004).

[9] Jun-Young Lee, Sangkook Choi, and Sang-Koog Kim, J. Magnetics 11, 74 (2006).

[10] Kab-Jin Kim, Chun-Yeol You, and Sug-Bong Choe, J. Magnetics 13, 136 (2008).

[11] Seung-Ho Kim, Han-Seok Lee, Seung-Hyun Lee, WooYoung Lee, J. Lee, J. Magnetics 13, 167 (2008).

[12] S. M. Cherif, Y. Roussigne, C. Dugautier, and P. Moch, J. Magn. Magn. Mater. 222, 337 (2000).

[13] S. Goolaup, A. O. Adeyeye, N. Singh, and G. Gubbiotti, Phys. Rev. B. 75, 144430 (2007). 